\title{
NUCLEOSYNTHESIS DURING THE MERGER OF WHITE DWARFS AND THE ORIGIN OF R CORONAE BOREALIS STARS
}

\author{
R. Longland ${ }^{1,2}$, P. Lorén-Aguilar ${ }^{2,3}$, J. José ${ }^{1,2}$, E. García-Berro ${ }^{2,3}$, L. G. Althaus ${ }^{4}$, And J. Isern ${ }^{2,5}$ \\ ${ }^{1}$ Departament de Física i Enginyeria Nuclear, EUETIB, Universitat Politècnica de Catalunya, c/Comte d'Urgell 187, \\ E-08036 Barcelona, Spain; richard.longland@upc.edu \\ 2 Institut d'Estudis Espacials de Catalunya (IEEC), Ed. Nexus-201, C/Gran Capità 2-4, E-08034 Barcelona, Spain \\ ${ }^{3}$ Departament de Física Aplicada, Universitat Politècnica de Catalunya, c/Esteve Terrades, 5, E-08860 Castelldefels, Spain \\ ${ }^{4}$ Facultad de Ciencias Astronómicas y Geofísicas, Universidad Nacional de La Plata, Paseo del Bosque s/n, (1900) La Plata, Argentina \\ 5 Institut de Ciències de 1'Espai (CSIC), Campus UAB, E-08193 Bellaterra, Spain \\ Received 2011 June 2; accepted 2011 June 30; published 2011 August 2
}

\begin{abstract}
Many hydrogen-deficient stars are characterized by surface abundance patterns that are hard to reconcile with conventional stellar evolution. Instead, it has been suggested that they may represent the result of a merger episode between a helium and a carbon-oxygen white dwarf. In this Letter, we present a nucleosynthesis study of the merger of a $0.4 M_{\odot}$ helium white dwarf with a $0.8 M_{\odot}$ carbon-oxygen white dwarf, by coupling the thermodynamic history of Smoothed Particle Hydrodynamics particles with a post-processing code. The resulting chemical abundance pattern, particularly for oxygen and fluorine, is in qualitative agreement with the observed abundances in R Coronae Borealis stars.
\end{abstract}

Key words: nuclear reactions, nucleosynthesis, abundances - stars: AGB and post-AGB - white dwarfs

Online-only material: color figures

\section{INTRODUCTION}

Hydrogen-deficient stars with high carbon abundances (with the exceptions of the PG 1159 and CSPN WR stars) can be divided into three main categories according to their effective temperatures: hydrogen-deficient carbon $(\mathrm{HdC})$ stars, R Coronae Borealis (RCB), and extreme helium (EHe) stars. They are often considered to be at different evolutionary stages following a common origin, as evidenced by their similar atmospheric abundances (Pandey et al. 2004).

The origin of these stars is of particular interest because of their unique chemical composition. As well as being enriched in carbon and oxygen relative to solar abundances, they also exhibit enrichment in nitrogen, neon, fluorine, and some $s$-processed material. These abundance patterns are hard to reconcile with their expected initial composition, requiring alternative scenarios for their origin. Two leading theories are the Final Flash (FF) and the Double-Degenerate (DD) scenarios. The FF scenario attributes the formation to a late He-shell flash in a post-asymptotic giant branch (post-AGB) star as it evolves toward the white dwarf state. The flash would move the star back onto the AGB part of the Hertzsprung-Russell diagram, while mixing $s$-processed material and He-burning ashes to the surface (Fujimoto 1977; Renzini 1979; Werner \& Herwig 2006). In the DD scenario, the merger of two white dwarfs $(\mathrm{He}+\mathrm{CO})$, with a total mass below the Chandrasekhar limit, through disruption and accretion of the lighter star, gives rise to a single object whose composition is an admixture of the two white dwarfs (Webbink 1984; Asplund et al. 2000). This dense object is expected to be surrounded by a hot corona and an accretion disk (Lorén-Aguilar et al. 2009). Surface abundance analyses of hydrogen-deficient stars seem to favor the latter scenario (Saio \& Jeffery 2002), although the possibility of some objects being formed through the FF scenario cannot be excluded (Pandey \& Lambert 2011).

When considering the formation of hydrogen-deficient objects through the DD scenario, the following question can be raised: does nuclear processing occur during the merging event? If no nuclear burning occurs- the "cold" merger hypothesis - the observed abundances might be reproduced through the partial mixing of material previously processed during the AGB phase of the $\mathrm{CO}$ white dwarf progenitor. However, recent calculations based on this scenario failed to qualitatively match the overall abundance pattern observed (Jeffery et al. 2011). Another possibility involves a "hot" merger event, in which significant nuclear processing occurs. Indeed, Clayton et al. (2007) suggested that this scenario may account for the high abundances of ${ }^{18} \mathrm{O}$ observed in some stars. Unfortunately, no detailed nucleosynthesis study during white dwarf mergers has been published to date.

In this Letter, we present a detailed study of the nucleosynthesis accompanying white dwarf mergers. Calculations are based on post-processing analyses using temperature and density versus time profiles obtained from smoothed particle hydrodynamics (SPH) simulations of the merger event. The structure of the manuscript is as follows. In Section 2 we summarize the observed abundances in hydrogen-deficient objects. The details of previous nucleosynthesis studies and of the calculations presented in this work are given in Sections 3 and 4, respectively. Results are discussed in Section 5, and the main conclusions are reached in Section 6.

\section{ABUNDANCES IN H-DEFICIENT STARS}

All current observed surface abundances of hydrogendeficient stars have been collected recently by Jeffery et al. $(2011)^{6}$, to which the reader is referred for details. Here we summarize the main observational features reported in that work.

Carbon is enriched in all observed hydrogen-deficient stars, irrespective of their iron content, with overabundances relative to solar of the order of 0.7 dex. Additionally, the inferred ${ }^{12} \mathrm{C} /{ }^{13} \mathrm{C}$ ratios are very large $(>500)$, indicating helium burning in the star. Nitrogen is also enriched in most EHe and RCB

With the exception of ${ }^{12} \mathrm{C} /{ }^{13} \mathrm{C}$ and ${ }^{16} \mathrm{O} /{ }^{18} \mathrm{O}$ ratios, and abundances of $\mathrm{Sc}$, $\mathrm{Mn}, \mathrm{Co}, \mathrm{Cu}$, and $\mathrm{Sr}$. 
stars. However, in this case it is correlated to the iron abundance, suggesting that nitrogen is enriched only by $\mathrm{CNO}$ cycling prior to the merging event, as can be shown by summing the initial carbon, oxygen, and nitrogen abundances of the star. Oxygen poses an interesting problem since its abundance exhibits a large spread. Regardless of this fact, oxygen appears to be both enriched and independent of metallicity. To account for the overabundance of oxygen a dredge-up event, by which oxygen is injected from the $\mathrm{CO}$ white dwarf, seems to be required. Because of the difficulties in distinguishing between isotopes in hotter stars, oxygen could also be produced in the form of ${ }^{18} \mathrm{O}$, which has been found to be highly enriched in a few stars (Clayton et al. 2007). Fluorine, in turn, is highly enriched by $2-4$ dex in all the observed stars. This enrichment could possibly arise from processing during the AGB phase of the $\mathrm{CO}$ white dwarf progenitor through a rather complicated process in the helium inter-shell zone (Lugaro et al. 2004). Finally, s-process elements, particularly yttrium and zirconium, have also been observed to be enriched by up to a factor of 50 in some EHe stars Pandey et al. (2004). The source of these elements is probably dredge-up of helium inter-shell products of the AGB star that subsequently evolved into the $\mathrm{CO}$ white dwarf. While several models can match some of these observational features to an order-of-magnitude level, the current challenge is to reproduce the overall abundance pattern simultaneously with a detailed model of the merger event.

\section{PREVIOUS NUCLEOSYNTHESIS STUDIES}

Most abundance studies of white dwarf mergers performed so far (Saio \& Jeffery 2002; Pandey \& Lambert 2011; Jeffery et al. 2011) consider the cold merger paradigm, in which no nucleosynthesis occurs. In particular, the recent work by Jeffery et al. (2011) started with the abundances present in the white dwarfs as resulting from previous evolution, particularly the AGB star evolution of the $\mathrm{CO}$ white dwarf progenitor. Calculations also included helium and hydrogen buffers on the surfaces of the white dwarfs, remaining from previous evolution, which contained material that had been heavily processed. By using a recipe for mixing the material in the white dwarfs during the merger, they were able to account for most of the abundances (or at least the trends) observed in EHe and RCB stars. The most notable exception, however, was the abundance of fluorine. Although fluorine can exist in the helium buffer of the $\mathrm{CO}$ white dwarf, it was found not to be abundant enough to account for the very high observed values.

Hot merger nucleosynthesis calculations were performed by Clayton et al. (2007) upon the discovery of very low ${ }^{16} \mathrm{O} /{ }^{18} \mathrm{O}$ ratios in some HdC and RCB stars. These studies relied on a one-zone parametric model to simulate the conditions needed to burn helium during a merger event. Their simple model was able to account for the enrichment of ${ }^{18} \mathrm{O}$ in these environments. When an admixture of hydrogen was added, however, the ${ }^{18} \mathrm{O}$ was rapidly destroyed through proton captures while ${ }^{16} \mathrm{O}$ was created through the ${ }^{13} \mathrm{C}(\alpha, n){ }^{16} \mathrm{O}$ reaction, thus restoring the ${ }^{16} \mathrm{O} /{ }^{18} \mathrm{O}$ ratio closer to solar values. Hot merger nucleosynthesis was also studied by Jeffery et al. (2011) in the absence of mixing of material from the $\mathrm{CO}$ white dwarf. They were able to account for overabundances in carbon and oxygen, but limitations in the SPH calculations provided by Lorén-Aguilar et al. (2009) prevented them from making reliable predictions for other nuclei. Detailed nucleosynthesis studies using realistic hydrodynamic models are therefore required to fully understand the production of elements in these environments.

\section{INITIAL MODELS AND INPUT PHYSICS}

The models used as input for the nucleosynthesis calculations reported in this Letter have been presented elsewhere (Guerrero et al. 2004; Lorén-Aguilar et al. 2009, 2010), and rely on an SPH Lagrangian simulation to follow the evolution of two coalescing white dwarfs. We refer the reader to these works for the details of the dynamical behavior of the merger process, but we describe here the structure of the final remnant, which is relevant for the calculations reported below. In essence, the final remnant of the merger process consists of a central hot white dwarf which contains most of the mass of the primary. On top of it a hot corona can be found, which contains most of the mass of the disrupted secondary and a small admixture of the primary white dwarf. Finally, surrounding the compact remnant, a rapidly rotating Keplerian disk is formed. It is also worth noting that little mass is ejected from the system. We emphasize that, for the sake of conciseness, we only describe the results obtained when the masses of the merging white dwarfs are 0.4 and $0.8 M_{\odot}$ with SPH particle masses of $2.6 \times 10^{-6} M_{\odot}$ and $5.2 \times 10^{-6} M_{\odot}$, respectively.

A network containing 14 nuclei was employed in the SPH simulations to account for the energy released by nuclear reactions during the merger (mostly $\alpha$-capture reactions), while the detailed nucleosynthesis reported here is calculated for tracer particles using a 327 nucleus post-processing network ranging from hydrogen to gallium. Energy generation rates between the two networks differs by less than $1 \%$ for our models. The thermonuclear reaction rates are adopted from the REACLIB database (Cyburt et al. 2010) with updates on experimental rates from Iliadis et al. (2010). SPH particles that reside in the hot corona of the final object will characterize the "atmosphere" that is observed in a hydrogen-deficient star. The tracer particles used to follow the thermodynamic history of the merger are therefore picked from $0.005<R / R_{\odot}<0.05$ as shown in Figure 1. This range includes particles that represent everything from the surface of the central dense object to the inner edge of the accretion disk. A total of 10,000 tracer particles have been used here to ensure a representative sample from the two white dwarfs.

Our simulations take into account the existence of thin helium and hydrogen buffer shells, which are predicted to survive prior evolution through the common envelope stage. Therefore, three different regions (carbon/oxygen-, helium-, and hydrogen-rich) are defined in the $\mathrm{CO}$ white dwarf, while two (helium- and hydrogen-rich) are adopted for the He white dwarf. These regions are defined according to the mass contained. Adopting the notation of Saio \& Jeffery (2002)—who, for instance, referred to the mass of the $\mathrm{He}$ shell in a $\mathrm{CO}$ white dwarf as $M_{\mathrm{He}: \mathrm{CO}}$-the different shells are defined as follows: $M_{\mathrm{CO}: \mathrm{CO}}=0.78, M_{\mathrm{He}: \mathrm{CO}}=0.019, M_{\mathrm{H}: \mathrm{CO}}=0.001$, $M_{\mathrm{He}: \mathrm{He}}=0.399$, and $M_{\mathrm{H}: \mathrm{He}}=0.001$. The chemical composition of each shell has been taken from detailed pre-white dwarf models (Renedo et al. 2010) of the appropriate metallicity, with some additional information from Jeffery et al. (2011) for the hydrogen buffers. It consists of seven species: ${ }^{1} \mathrm{H},{ }^{4} \mathrm{He},{ }^{12} \mathrm{C},{ }^{14} \mathrm{~N},{ }^{16} \mathrm{O},{ }^{17} \mathrm{O}$, and ${ }^{22} \mathrm{Ne}$. It is worth noting that, although we do not expect to reproduce the observed $s$-process abundances in hydrogen-deficient stars with only these seven isotopes, they should be enough to model nucleosynthesis of light and intermediate-mass elements (up to neon). Two metallicity scenarios have been considered: (1) solar metallicity and (2) a lower metallicity one, with $Z=10^{-5}$. 


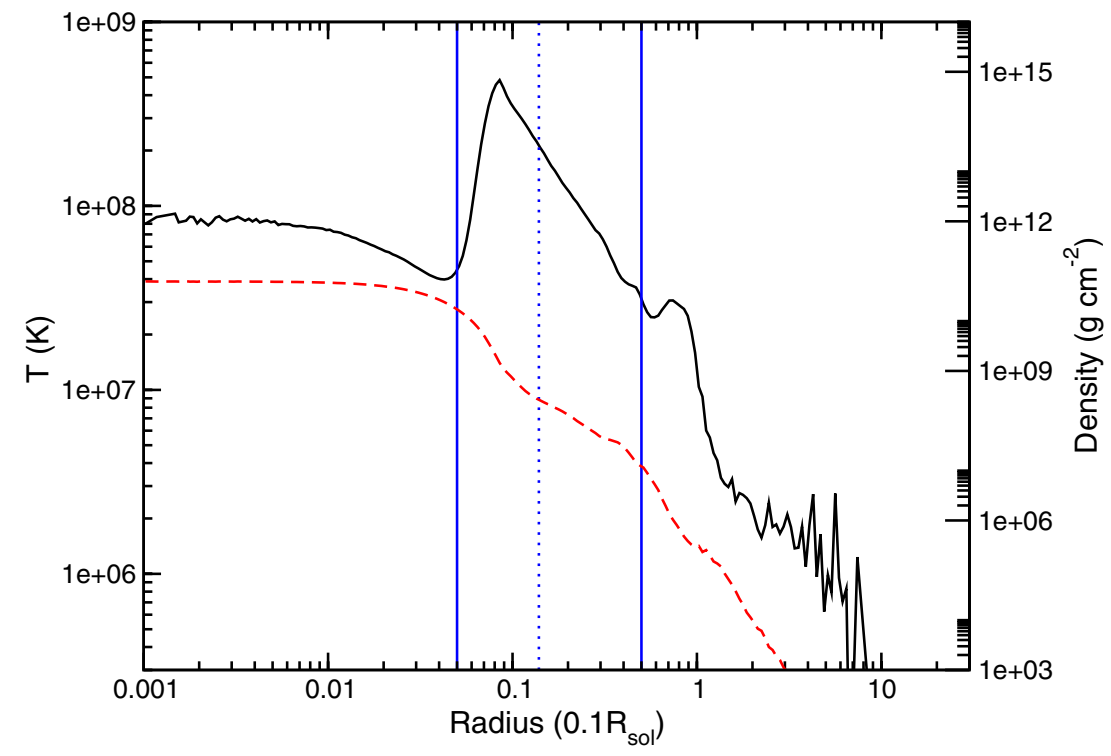

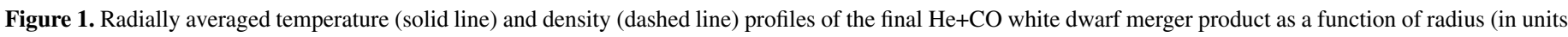

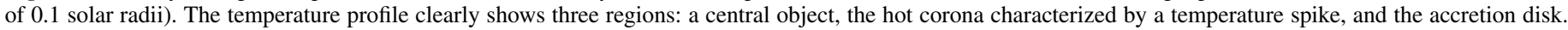
The vertical lines indicate the limit of the convective mixing region (solid representing "deep" mixing and dotted for "shallow" mixing in the hot corona).

(A color version of this figure is available in the online journal.)

To account for convective mixing of the material in the hot corona, we calculate the mass-averaged abundances of all particles that reside within a defined convective zone at the end of the hydrodynamic simulation. Although the SPH calculations suggest that the entire hot corona will be convective, we adjust the depth of the mixing in our analysis to study the effect of this assumption on the final surface abundances. Here, we consider two cases: (1) "deep" mixing, in which everything in the range $0.005<R / R_{\odot}<0.05$ is mixed homogeneously and (2) "shallow" mixing, in which the range is assumed to be smaller, $0.014<R / R_{\odot}<0.05$.

\section{RESULTS AND DISCUSSION}

The mass-averaged abundances obtained from the tracer particle nucleosynthesis are compared with the observed abundances in Figure 2. The plotted solid lines correspond to the final abundances obtained under the "deep" mixing assumption, while surface abundances obtained assuming a "shallow" mixing depth are represented by dashed lines. These lines are added purely to guide the eye since our calculations were only performed for two metallicity cases $\left(z=0.015\right.$ and $\left.z=10^{-5}\right)$. In judging the successfulness of our models, it is worthwhile to compare each element to observed abundances on a one by one basis.

Carbon depends strongly on the mixing depth assumed in the hot corona of the merger product but appears to be consistently high in our calculations. Nevertheless, the overall trend of carbon abundance with respect to metallicity agrees with the observational data. The ${ }^{13} \mathrm{C} /{ }^{12} \mathrm{C}$ ratio for solar metallicity is $\sim 2 \times 10^{-5}$, consistent with the predicted nature of heliumburned material, but considerably higher than the observed ratio. This disagreement could arise from the limited number of nuclear species adopted in our calculations.

The oxygen abundance also agrees fairly well with the observed data, showing a large range of values depending on the mixing depth assumed. This dependence could explain the observed scatter in oxygen in EHe and RCB stars, where the observed oxygen abundance could depend strongly on the nature of the individual merging event. This picture is not, however, consistent with the relatively low scatter in carbon abundances. With regard to the isotopic abundances, our solar metallicity model for deep and shallow mixing yields ${ }^{16} \mathrm{O} /{ }^{18} \mathrm{O}=370$ and 19 , respectively. The ${ }^{18} \mathrm{O}$ has clearly been enhanced in the outer regions of the hot corona with respect to solar abundances (where ${ }^{16} \mathrm{O} /{ }^{18} \mathrm{O} \approx 400$ ), although not to the extent found in some HdC and RCB stars (Clayton et al. 2007).

Nitrogen follows the expected trend indicating that it is enhanced only through CNO cycling in the parent stars. Although it is slightly reduced by the ${ }^{14} \mathrm{~N}(\alpha, \gamma){ }^{18} \mathrm{~F}$ reaction, it is present in such a high concentration that it is barely diminished in the short timescales involved.

The overabundances of fluorine obtained in our models are high, but not high enough to fully agree with the observational data. The lack of sensitivity to mixing depth is an indication that fluorine is produced homogeneously in the merging event, and not in any particular region of the merger. Fluorine is produced dominantly through the following sequence of reactions: ${ }^{14} \mathrm{~N}(\alpha, \gamma){ }^{18} \mathrm{~F}(\mathrm{p}, \alpha){ }^{15} \mathrm{O}(\alpha, \gamma){ }^{19} \mathrm{Ne}\left(\beta^{+}\right){ }^{19} \mathrm{~F}$. Enrichment of fluorine in our models is a great success, suggesting that hydrogendeficient stars could be the result of a hot white dwarf merger event since it is challenging to reconcile those high abundances with a cold merger event.

Finally, the abundance of neon following the merger is consistent with the metallicity, indicating that it is not processed in either the merger event, or during the preceding evolution of the parent stars. Neither our models nor the cold merger models of Jeffery et al. (2011) reproduce the observed neon abundance in several EHe stars, which contain overabundances up to about 1 dex. Clearly more work must be performed to understand the nucleosynthesis of neon in hydrogen-deficient stars.

In summary, our models of hot white dwarf mergers are able to reproduce, on an order-of-magnitude level, the surface abundances of light and intermediate-mass nuclei in hydrogen- 


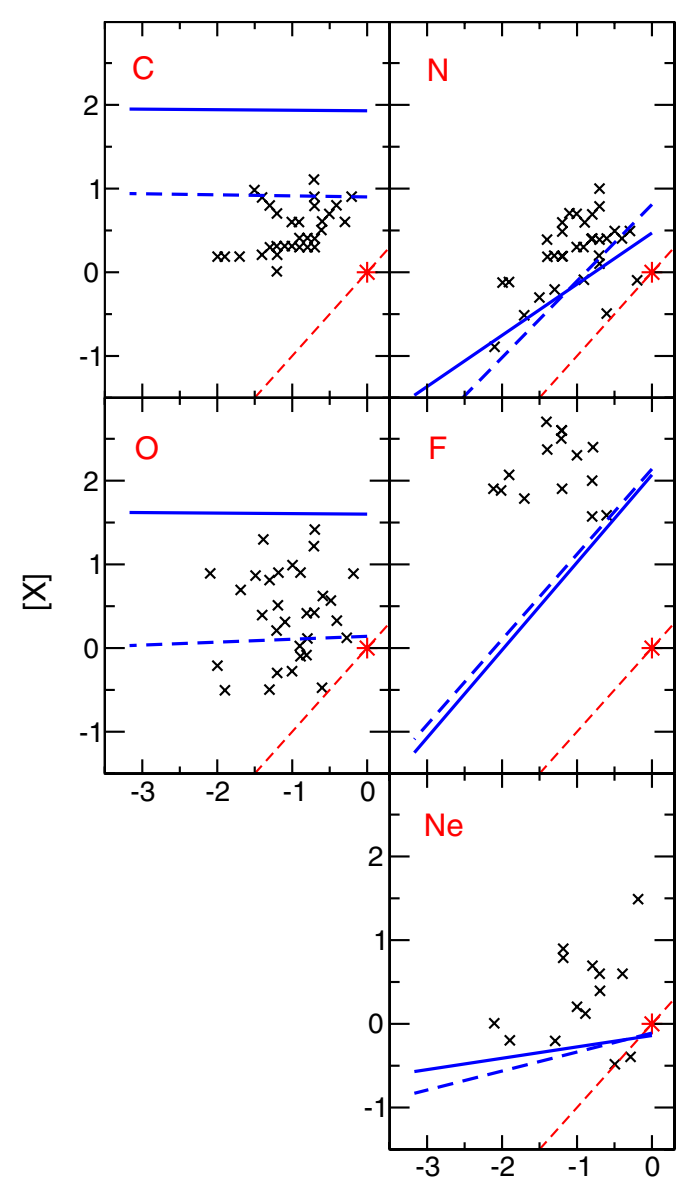

$[\mathrm{Fe}]$

Figure 2. Hydrogen-deficient star surface abundance determinations taken from Jeffery et al. (2011) compared to our nucleosynthesis yields computed in the merger of a $0.4 M_{\odot}$ and a $0.8 M_{\odot}$ white dwarfs. The axis labels, [X] and [Fe], correspond to the logarithmic abundances relative to solar for individual elements and for iron, respectively. Solid lines are our calculations assuming "deep" convective mixing, while dashed lines are for "shallow" mixing (see the text). The lines are added to guide the eye since our calculations include only two metallicities: $Z=0.015$ and $Z=10^{-5}$. The asterisk symbol corresponds to solar abundances, while the diagonal lines intersecting solar values are the abundances expected if the solar values were scaled with metallicity.

(A color version of this figure is available in the online journal.)

deficient stars. The model's main success is in its ability to synthesize high fluorine abundances, which cannot be produced in cold merger models (Jeffery et al. 2011).

\section{CONCLUSIONS}

Mergers of helium and carbon-oxygen white dwarfs are currently favored as the events responsible for the production of hydrogen-deficient $\mathrm{HdC}, \mathrm{EHe}$, and RCB stars. The main question facing us is whether these objects are the result of a cold merger, in which no nucleosynthesis occurs, or a hot merger, in which at least some of the material is processed through nucleosynthesis.

The results presented in this work show that nuclear processing during the merger event can account for some of the observed abundances in these stars, particularly for fluorine, which cannot be easily synthesized in the cold merger scenario. There are, however, still open questions regarding the expected nucleosynthesis: neon is difficult to produce at the observed levels, while the absolute abundance of carbon is too high in our models. Such discrepancies may be alleviated by the inclusion of a more detailed initial chemical composition with many more species, resulting from state-of-the-art models of the preceding AGB phase. Inclusion of such detailed abundances is currently under way.

This work has been partially supported by the Spanish grants AYA2010-15685, AYA08-1839/ESP, and AYA2008- 04211C02-01, by the E.U. FEDER funds, and by the ESF EUROCORES Program EuroGENESIS through the MICINN grants EUI2009-04167 and 04170.

\section{REFERENCES}

Asplund, M., Gustafsson, B., Lambert, D. L., \& Rao, N. K. 2000, A\&A, 353, 287

Clayton, G. C., Geballe, T. R., Herwig, F., Fryer, C., \& Asplund, M. 2007, ApJ, 662, 1220

Cyburt, R. H., Amthor, A. M., Ferguson, R., et al. 2010, ApJS, 189, 240

Fujimoto, M. Y. 1977, PASJ, 29, 331

Guerrero, J., García-Berro, E., \& Isern, J. 2004, A\&A, 413, 257

Iliadis, C., Longland, R., Champagne, A. E., Coc, A., \& Fitzgerald, R. 2010, Nucl. Phys. A, 841, 31

Jeffery, C. S., Karakas, A. I., \& Saio, H. 2011, MNRAS, 414, 3599

Lorén-Aguilar, P., Isern, J., \& García-Berro, E. 2009, A\&A, 500, 1193

Lorén-Aguilar, P., Isern, J., \& García-Berro, E. 2010, MNRAS, 406, 2749

Lugaro, M., Ugalde, C., Karakas, A. I., et al. 2004, ApJ, 615, 934

Pandey, G., \& Lambert, D. L. 2011, ApJ, 727, 122

Pandey, G., Lambert, D. L., Rao, N. K., \& Jeffery, C. S. 2004, ApJ, 602, L113

Renedo, I., Althaus, L. G., Miller Bertolami, M. M., et al. 2010, ApJ, 717, 183

Renzini, A. 1979, in Stars and Star Systems, ed. B. E. Westerlund (Astrophysics and Space Science Library, Vol. 75; Dordrecht: Reidel), 155

Saio, H., \& Jeffery, C. S. 2002, MNRAS, 333, 121

Webbink, R. F. 1984, ApJ, 277, 355

Werner, K., \& Herwig, F. 2006, PASP, 118, 183 